\title{
The Distribution of Cytoplasmic and Nuclear NF-кB in IL-1b Activated Cardiac Fibroblasts is Non-Random Relative to the $Z$ topology of the Nucleus.
}

\author{
J.W. Fuseler and W. Carver \\ University of South Carolina School of Medicine \\ Department of Cell Biology and Anatomy \\ Columbia, South Carolina 29209
}

Nuclear factor-kappa B (NF- $\kappa \mathrm{B})$ is a heterodimeric transcription factor typically composed of p50 and p65 subunits and is known to be a pleiotropic regulator of various inflammatory and immune responses. In unstimulated cells, p50/p65 dimers are sequestered in the cytoplasm via interaction with specific inhibitor proteins, the I- $\kappa$ Bs. Upon cellular activation, the I- $\kappa$ Bs are proteolytically degraded, releasing the active form of NF- $\kappa \mathrm{B}$ (p65/p50 heterodimers). The active form of NF- $\kappa \mathrm{B}$ is rapidly translocated into the nucleus, reaching a maximum by 30 minutes, where it binds to its consensus sequences in the promoter/enhancer region of various genes and subsequently promotes transcription of these genes. NF- $\kappa \mathrm{B}$ then undergoes inactivation and moves back into the cytoplasm becoming minimally present in the nucleus within 2 hours [1]. Here we demonstrate that the distribution of NF- $\mathrm{KB}$ in the cytoplasm and nucleoplasm of quiescent and cytokine stimulated cardiac fibroblasts is not random but localized in specific regions.

Primary cultured embryonic rat cardiac fibroblasts (CF) were grown on collagen coated $22 \times 22$ mm cover slips and used at approximately $80 \%$ confluent. CF were activated with Rat IL-1b $(10 \mathrm{ng} / \mathrm{ml})$ and fixed at time points from 0 (negative control) to 60 minutes. The localization of the p65 subunit of NF- $\kappa \mathrm{B}(\mathrm{p} 65-\mathrm{NF}-\kappa \mathrm{B})$ in the nucleus and cytoplasm was visualized and quantified by immunocytochemisrty $[1,2]$. The nucleus was labeled with DAPI. The distribution of p65-NF- $\kappa \mathrm{B}$ in the labeled cells was determined by confocal microscopy in which images of the CF were collected at 1 um sections in the Z-plane. Quantification of p65-NF- $\kappa B$ in cytoplasmic and nuclear Z-planes of the CF was determined by measurement of its fluorescence integrated optical density (IOD) by image analysis and it state of its organization determined by calculation of its fractal dimension (D)[3]. The IOD and D for p65-NF- $\mathrm{kB}$ in the cytoplasm and nucleus were correlated with the area of the nucleus present in the same Z-plane.

In quiescent $\mathrm{CF}, \mathrm{p} 65-\mathrm{NF}-\kappa \mathrm{B}$ is predominantly localized in the cytoplasm with only a minimal presence in the nucleus. In the cytoplasm, the p65-NF- $\mathrm{BB}$ is predominantly localized in a narrow $2 \mathrm{um}$ plane located below the major area and diameter (equator) of the nucleus. The small of amount of p65-NF- $\mathrm{kB}$ present in the quiescent nucleus is punctate and located predominantly with the nuclear margin. The $\mathbf{D}$ value of the nuclear $\mathrm{p} 65-\mathrm{NF}-\kappa \mathrm{B}$ is small, invariably being less 
than 1.0 (mean of $0.874 \pm 0.112$ ). This suggests that the distribution of $\mathrm{p} 65-\mathrm{NF}-\kappa \mathrm{B}$ in the quiescent nuclear Z-planes has a distribution which resembles that of Cantor dust set.

Following 20-30 minutes of activation by IL-1b, p65-NF- $\kappa$ B reaches maximal localization in the nucleus. In activated cells, the cytoplasmic p65-NF- $\mathrm{B}$ remains localized below the equator of the nucleus, with the IOD reduced to about half that seen in the negative controls. In the nucleus, p65-NF- $\mathrm{kB}$ appears localized as unequal peaks of IOD with the major peak localized in the lower regions of the nucleus with minimal p65-NF- $\kappa \mathrm{B}$ present at the nuclear equator. The mean p65-NF- $\kappa$ B nuclear IOD at this time point is $213.53 \pm 36.59$ and the mean $\mathbf{D}$ value is 1.440 \pm 0.078 . This indicates that activated nuclear $\mathrm{p} 65-\mathrm{NF}-\kappa \mathrm{B}$ associated with its DNA consensus sequences is highly ordered and moderately space filling.

At 40 minutes following activation by IL-1b, during translocation of NF- $\kappa B$ back into the cytoplasm the major nuclear IOD peak of p65-NF- $\kappa \mathrm{B}$ shifts to the upper latitudes above the

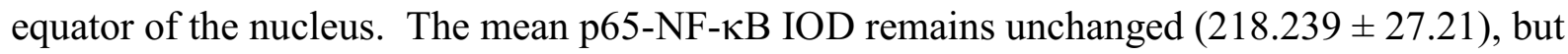
there is significant increase in the mean $\mathbf{D}$ value (1.701 \pm 0.039$)$. This increase in $\mathbf{D}$ suggests inactivation and movements associated with export translocation of p65-NF- $\kappa$ B out of the nucleus is characterized by a significant increase in chaos indicating the complexes containing the p65 subunits have under gone increased randomization and have become more space filling.

These data suggest that cytoplasmic NF- $\kappa \mathrm{B}$ in quiescent cardiac fibroblasts is not randomly distributed throughout the cytoplasm but is predominately localized in a specific narrow region relative to the equator of the nucleus. These data further indicate that following activation and translocation into the nucleus, NF- $\mathrm{KB}$ is also not randomly distributed, but localized to specific regions of the nucleoplasm when in association with its specific DNA consensus-sequence targets. During the early stages of export, the increased $\mathbf{D}$ values associated with inactivation and export of NF- $\mathrm{KB}$ out of the nucleus suggest that these events are characterized by disorganization and an increase in chaos associated with the NF- $\kappa \mathrm{B}$ complex.

\section{References;}

1.Rogers, J.A., Fuseler, J.W. 2007. Regulation of NF-кB activation and nuclear translocation by exogenous nitric oxide (NO) donors in TNF- $\alpha$ activated vascular endothelial cells. Nitric Oxide. 16: 379-391.

2.Fuseler J.W. Merrill D.M., Rogers J.A. Grisham M.B. Wolf R.E. 2006. Analysis and quantitation of NF- $\kappa \mathrm{B}$ nuclear translocation in tumor necrosis factor alpha activated vascular endothelial cells. Microsc. Microanal.12: 269-276.

3.Fuseler, J.W., Millette, C.F., Davis, J.M., Wayne Carver, W. 2007. Fractal and image analysis of morphological changes in the actin cytoskeleton of neonatal cardiac fibroblasts in response to mechanical stretch. Microsc. Microanal.13: 128-132. 